\title{
Adaptive Playout Strategies for Packet Video Receivers with Finite Buffer Capacity
}

\author{
Nikolaos Laoutaris and Ioannis Stavrakakis \\ Department of Informatics, University of Athens, 15784 Athens, Greece \\ \{laoutaris,istavrak\}@di.uoa.gr
}

\begin{abstract}
Due to random delay variations in current best effort networks, packet video applications rely on end-system buffering and playout adaptation to reduce the effects of disruptions on the required smooth stream presentation. To study the effect of buffering and playout adaptation, we present an analytical model based on the M/G/1 queueing system with finite buffer capacity and traffic intensity equal to or greater than unity. This model fits well a range of new applications that have limited buffer resources for the reception of incoming frames. We introduce the Variance of Distortion of Playout (VDoP), a new metric that accounts for the overall presentation disruption caused by buffer underflows, intentionally introduced gaps during slowdown periods and data loss from overflows. VDoP is an elegant and fair metric for the estimation of playout quality and will hopefully assist the development of better adaptation algorithms. Furthermore, the effect of finite buffer capacity is examined in relation to stream continuity, revealing a system behavior not previously accounted for. The sensitivity of the system to the variance of the arrival process is also examined by means of simulation. Finally, an online algorithm is presented for the exploitation of our study on implemented systems.
\end{abstract}

Keywords-Adaptive video playout, video continuity, finite M/G/1, Markov Modulated Poisson Process

\section{INTRODUCTION}

In recent years multimedia services such as internet telephony, videoconference, and video on demand (VOD), have found a place next to traditional data applications like telnet, ftp or the world wide web. These new services require high transmission reliability and stringent end-to-end delay and delay jitter, to be able to maintain interstream synchronization between successive media units. The main effort in providing current best effort networks with QoS mechanism has been undertaken by the IETF which is standardizing the Integrated Services and Differentiated Services architectures. Nevertheless, it is realized that the deployment of new protocols will be a slow process, so much effort is being put in coping with current network limitations by incorporating intelligent adaptive algorithms at the application layer.

Adaptive rate applications fall into two general categories depending on which end of the communicating parties is adapting its rate. In source rate adaptation [1], [2], it is the sending system that adapts to the time-varying bandwidth availability by regulating the rate of its output video stream. On the other hand, Packet Video Receiving systems (PVRs), adapt their playout rate in an effort to avoid underflows (lack of a frame to display) caused by excessively delayed frames [3], [4], [5]. PVRs use a playout buffer to accumulate incoming frames as a measure against network jitter. The amount of buffered frames can be dynamically adjusted in response to network jitter. When the variability of the arrival process increases, the PVR must buffer more frames, increasing the end-to-end latency, to avoid large underflows. If there is a latency constraint, then in times when network load reduces and in-

This work and its dissemination efforts have been supported in part by the General Secretariat for Research and Development of Greece under grants 99PENED92 and 99PENED-193 and the IST Program of the European Union under contact IST-1999-10160 (VideoGateway). terframe spacing is not distorted by varying end-to-end delays, the system can decide to reduce the amount of buffered frames, thus gain in end-to-end delay reduction. The playout buffer occupancy increases when the continuous presentation of frames is disturbed by idle time gaps, for example underflows. Underflows seriously degrade video quality as they can last for a considerable amount of time. Adaptive playout PVRs try to reduce the frequency of underflows by intentionally introducing virtual time gaps between successive frames, when the buffer level is too low. If these virtual gaps are shorter than underflows, then they may be unnoticed due to human perceptual limitations. In principle, the annoyance caused by a large underflow is spread in time, so as to reduce its impact on video quality. Virtual time gaps are created by keeping the current frame on display longer than what the video rate suggests, in other words by displaying frames slower than their actual rate. This unfortunate necessity is not present in packet audio systems, where the existence of silence periods gives the system the ability to change the size of the de-jitter buffer by modifying the duration of the silence period in a per talkspurt basis without modifying the playout rate [6], [7], [8], [9].

Buffering frames at the PVR increases end-to-end latency at the end-user level. Different applications tolerate different maximum end-to-end latencies. Bidirectional applications such as desktop video conferencing applications, have very strict latency requirements, typically of few hundreds of milliseconds. On the other hand, unidirectional applications like VOD, allow for much larger latencies, in the order of seconds. In a VOD application, the PVR can buffer massive numbers of frames, thus ensure an almost pauseless video presentation across the widest range of network jitter. The absence of critical latency requirements also allows for the addition of techniques such as data proxy-ing and client-server feedback which can help in using network resources more efficiently, especially in the case of Variable Bit Rate (VBR) encoded video [10], [11], [12].

In this paper we consider a class of applications with a latency requirement that falls between the hard real-time video conferencing and the delay immune VOD. We would include in this category most Web-based Video Distribution (WVD) systems. Such video applications do not require a minimal end-to-end latency as in video conferencing, but cannot tolerate a large start up buffering delay as in VOD. The key differentiating factor from VOD, is the click level interactivity requirement, i.e., the requirement for a short time interval between a user video selection instant and the display instant of the first video frame. This requirement is largely due to small reaction times that most web based applications tend to provide. In other words, WVD systems require a small reaction time (so they cannot pre-buffer many frames) but are not sensitive to end-to-end latency over the entire duration of the presentation. The interactivity requirement suggests that video 
presentation starts after only a small number of frames has been buffered. If the initial de-jitter buffer is not adequate to smooth out network delay variabilities, the buffer progressively expands by applying reduced playout rates. A final note on our targeted application area is that the buffer space that they can utilize is limited. This is definitely the case for wide spread web based PVRs, designed even for the modest desktop system. It is also expected that PVRs with limited buffer space will be used in third generation wireless terminal devices.

\section{RELATED WORK}

In this section we provide a brief presentation on threshold based playout rate adaptation schemes. The main idea in threshold based rate adaptation is the linear reduction of playout rate as the number of buffered frames approaches zero. Yuang et al. [3] propose a PVR that employs a maximum playout rate $\mu$, when the number of buffered frames $i$ is greater than, or equal to, a given threshold value $\mathrm{TH}$, and employs reduced playout rates $\mu(i)=\mu \cdot i / \mathrm{TH}$ for $i<\mathrm{TH}$. The analysis in [3] models the PVR as a queueing system with Poisson arrivals of rate $\lambda$ and exponential service durations with state dependent mean service rate $\mu(i)$. It should be noticed that when the system operates above TH it employs a maximum playout rate $\mu$ which is greater than $\lambda$, the actual video frame rate. That is, the system displays frames faster than the normal video rate. Although the latter may leed to a latency reduction, it also produces an undesirable fast forward effect. Clearly, the value of TH impacts on the system performance. More specifically, the value of TH is examined in relation to the probability of an empty buffer (thus a continuity metric) $\pi_{0}$, the frame loss probability due to buffer overflow, $p_{L}$, and the mean presentation rate, $\bar{B}$. It is stated that both $\pi_{0}$ and $\bar{B}$ decrease with $\mathrm{TH}$, while $p_{L}$ increases with TH. The selection of TH can be seen as a tradeoff between the preservation of playout continuity, which is captured by $\pi_{0}$, and the reduction of mean playout rate with respect to the actual video rate, which is captured by $\bar{B}$. The continuity metric $\pi_{0}$, is replaced in [4] by the more general Variance of Discontinuity (VoD) metric, which not only captures the frequency of underflow gaps, but also accounts for gaps occurring due to the dynamic regulation of frame durations. In section III we simplify the study of a PVR by combining gap and loss metrics in a single continuity metric.

\section{SySTEM MODEL}

In this section we describe an analytical model for the study of a finite-buffer PVR, and examine the effect of TH on video quality. In what follows, we define the variance of discontinuity (VoD, as in [4]) and generalize it by introducing the variance of distortion of playout (VDoP). We use the analytical results to demonstrate the tradeoff between playout continuity and mean playout rate. Also, a new system behavior that affects the selection of $\mathrm{TH}$ is brought in light.

\section{A. Model Definitions and Assumptions}

We model the PVR as a finite queue with capacity for $N$ frames. The arrival process is Poisson with mean arrival rate $\lambda$, equal to the video frame rate $\mu$. Admittedly, the choice of an exponential distribution to model the frame interarrivals is a poor one, as indicated by internet delay measurements [13], [14]. Nevertheless, the exponential distribution provides for a large coefficient of variation, compared to actual interarrival distributions which fit better to a normal distribution, so it can be used as a lower bound on system performance. The exponential distribution models effectively the interarrivals when the network is overloaded and frames tend to arrive in clusters followed by large periods with no arrivals.

The playout controller of the system displays frames at a rate $\mu$ equal to the actual video rate $\lambda$, when $i$, the number of buffered frames, is greater than or equal to a threshold value TH. In all other cases it plays frames at a linearly declining rate $\mu(i)$ corresponding to an extended frame duration $B(i)$ according to:

$$
\begin{gathered}
\mu(i) \triangleq \begin{cases}\min \left(\frac{\mu \cdot i}{\mathrm{TH}}, \mu\right) & \text { if } 1 \leq i \leq N \\
\frac{\mu}{\mathrm{TH}} & \text { if } i=0\end{cases} \\
B(i) \triangleq \begin{cases}\max \left(\frac{\mathrm{TH}}{\mu \cdot i}, \frac{1}{\mu}\right) & \text { if } 1 \leq i \leq N \\
\frac{\mathrm{TH}}{\mu} & \text { if } i=0\end{cases}
\end{gathered}
$$

If $T=1 / \mu$ is the normal frame duration, keeping the frame on display for more than $T$, according to $B(i)$, is equivalent to introducing logical time discontinuities $d(i)$ between frames, during which no new frame is displayed as it should. The end user will realize $d(i)$ s as frame freezes. The duration of $d(i)$ is state dependent; for non-zero $i, d(i)$ is the difference $B(i)$ minus $T$. Thus,

$$
d(i) \triangleq \begin{cases}\max \left(\frac{\mathrm{TH}-i}{\mu \cdot i}, 0\right) & \text { if } \quad 1 \leq i \leq N \\ S+\frac{\mathrm{TH}-1}{\mu} & \text { if } i=0\end{cases}
$$

Note that $d(0)$ is composed of two terms, $S$ which is a random variable for the time interval from buffer underflow instant to the first new arrival instant, and $\frac{\mathrm{TH}-1}{\mu}$ which is the discontinuity $d(1)$, corresponding to the reduced playout rate when there is only one frame in the buffer ${ }^{1}$. In IV-A we use the exponential distributed $S$, with mean value $1 / \lambda$, for the derivation of the steady state behavior of the system.

In [4] the variance of discontinuity (VoD) is used as a stream continuity metric. VoD is just $\lim _{n \rightarrow \infty} E\left\{\left(d_{n}-\bar{d}\right)^{2}\right\}$, where $d_{n}$ is the discontinuity over the $n$th played frame and $\bar{d}$ is the expected value of $d$. VoD captures the disruptive effect of underflows and of intentionally introduced gaps. We enhance VoD by introducing the Variance of Distortion of Playout (VDoP), which adds to VoD the disruptive effect of frame losses due to buffer overflow. VDoP can also be used in systems where late frames are considered lost. VDoP captures the variance of the Distortion of Playout (DoP) which is caused either by interframe gaps or by loss gaps. We define the state dependent DoP as:

$$
D o P(i) \triangleq \alpha \cdot d(i)+\beta \cdot \bar{l}_{i} \cdot T
$$

$\overline{l_{i}}$ is the expected number of lost frames during the next frame playout, given that the system is left with $i$ frames just after the last frame presentation. $\alpha$ and $\beta$ are weights for jitter gaps and lost frames. Throughout the remainder of the paper we use equal weights $\alpha=\beta=1$. This selection is based on recent studies [15] that report an equal degradation of perceptual quality in the presence of jitter and packet loss. We define $l_{i, n}^{k}$ as:

\section{$l_{i, n}^{k} \triangleq \operatorname{Prob}\{k$ lost frames during playout of $n+1$ th frame $n$th played frame leaves the system in state $i\}$}

\footnotetext{
${ }^{1}$ Following an underflow, playout will start as soon as a frame arrives. The playout duration for that frame will be $B(1)$.
} 
$l_{i}^{k} \triangleq \lim _{n \rightarrow \infty} l_{i, n}^{k}$ is the steady state probability as $n$ approaches infinity. $l_{i}^{k}$ is given by:

$$
l_{i}^{k}=P\{N-i+1+k, B(i)\}
$$

where $P\{m, t\}$ is the Poisson distribution for the probability of $m$ new arrivals in an interval of duration $t$ and $N$ is the buffer size. $\overline{l_{i}}$ is the expected value of $l_{i}^{k}$.

The integration of all causes of discontinuity, to a single continuity metric, DoP, provides the common ground for the comparison of all playout adaptation schemes on the same basis. In the current work we have focused on threshold based rate adaptation, nevertheless, DoP can also be used to quantify the performance of more general adaptation schemes, e.g., schemes that not only expand the duration of frames but also shorten it to control latency.

\section{B. The M/G/l Queue and the Embedded Markov Chain}

In this section we derive the steady state probability distribution for $\left\{I_{n}\right\}_{n>0}$, the playout buffer occupancy on the $n$th frame departure instant (completion of the $n$th frame presentation). Under the Poisson assumption $\left\{I_{n}\right\}$ becomes a Markov chain. The state transition probabilities $p_{i, j}$ for the buffer occupancy chain are given by:

$$
p_{i, j}= \begin{cases}P\{j, B(0)\} & i=0,0 \leq j<N \\ 1-\sum_{k=0}^{N-1} P\{k, B(0)\} & i=0, j=N \\ P\{j-i+1, B(i)\} & 0<i<N, i \leq j<N \\ 1-\sum_{k=0}^{N-i} P\{k, B(i)\} & 0<i \leq N, j=N \\ P\{0, B(i)\} & 0<i \leq N, j=i-1 \\ 0 & \text { elsewhere }\end{cases}
$$

We use an iterative method for the solution of the stationary equations $\pi(i)^{(n+1)}=\pi(i)^{(n)} \cdot P$, where $\mathrm{P}$ is the transition matrix of (4) and $\pi(i), i=0, \ldots, N$, are the steady state probabilities of $\left\{I_{n}\right\}$. The superscript indicates the iteration step number.

\section{RESUlTS AND Discussion}

In this section we study the effect of TH on the two antagonistic metrics, the reduction of the Mean Playout Rate (MPR), with respect to the actual playout rate $\mu$, and the stream continuity metric. For stream continuity we use both VoD and VDoP and justify the advantages of the second over the first.

\section{A. Analytical Results}

The results are derived under the Poisson arrival process assumption and the forementioned M/G/1 formulation. MPR is the expected value of (1); VoD and VDoP, are the variances of (2), (3), respectively. In Fig. 1, we illustrate the effect of TH on a PVR with a buffer space for $N=100$ frames and $\lambda=\mu=30$ frames/second. The top graph illustrates the reduction of MPR with TH. This is an expected behavior as the scheduler employs reduced playout rates more frequently under a large $\mathrm{TH}$ value. In the middle graph we plot VoD against TH. This graph reveals an interesting system behavior. Contrary to what we would expect, increasing TH does not always lead to lower (better) values for VoD. This occurs because from a TH value and on, the mean occupancy of the buffer is relatively high compared to the maximum buffer capacity. This, in conjunction with the variability of the arrival process, causes substantial packet losses due to buffer overflow. Buffer overflows increase the frequency at which the occupancy drops below TH.
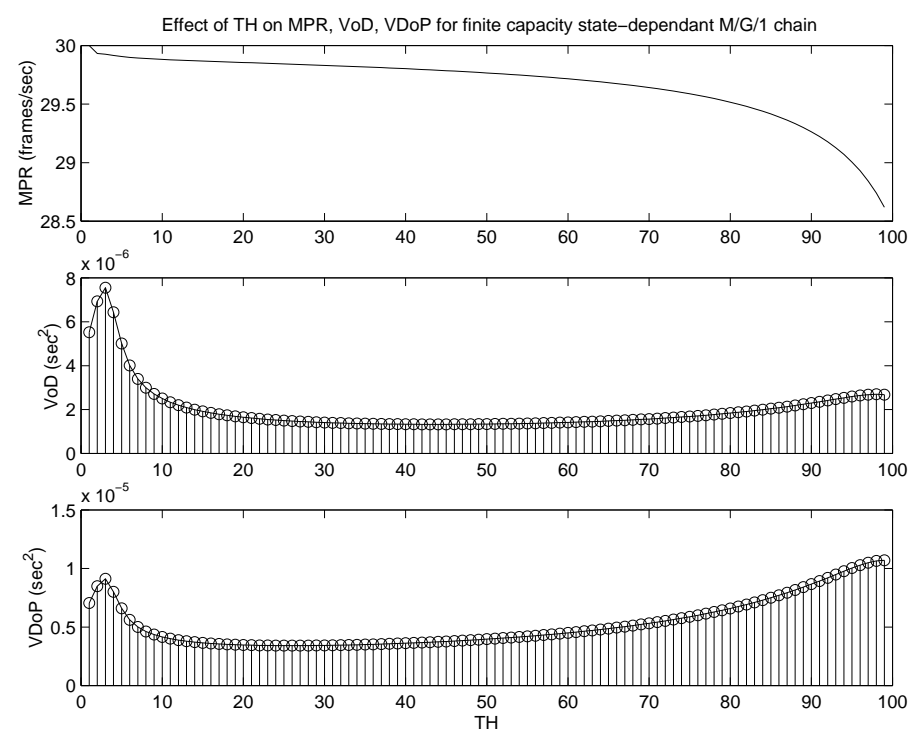

Fig. 1. Analytical results for a system with buffer for $N=100$ frames.

This in turn means that the playout controller has to apply a reduced playout rate not due to the arrival variability and the danger of underflow, but due to the massive overflows which drop the occupancy below TH. This is clearly an undesirable effect that limits the area where a healthy tradeoff between VoD and MPR exists. Moving to the right of that critical value of TH, results in a deterioration of both metrics of interest thus should definitely be avoided. The position of the critical TH value depends on the relationship between the variability of the arrival process and the size of the buffer. In Section IV-B we study this relationship by means of simulation using a two state ON-OFF Markov Modulated Poisson Process.

In the bottom graph of Fig. 1 we plot VDoP against TH. Comparing VDoP with VoD we recognize that both metrics have the same behavior with TH. The values of VDoP are higher than those of VoD for the same TH value. That is because VDoP adds to VoD the disruptive effect of overflowed frames. Moreover, we see that the monotonicity of the VDoP curve changes at some TH and is increasing aggressively as TH approaches the maximum buffer size. There are two reasons for this behavior. The first is the same as the one already discussed for $\mathrm{VoD}$, and has to do with slowdowns caused by the buffer occupancy reduction because of losses due to overflow. The second reason, not captured by VoD, is due to the rapid loss of overflowed frames when operating at high $\mathrm{TH}$ values.

\section{B. Simulation Results With MMPP Sources}

In this section we study the sensitivity of the playout rate adaptation mechanism to network congestion. Congestion creates jitter which is realized as burstiness of the frame arrival process. We have constructed a simulation model that employs an exponential ON-OFF Markov Modulated Poisson Process [16] for the generation of frame arrivals. By choosing appropriate parameters for the ON-OFF model, we create an arrival process that is more bursty than the Poisson process of equal rate. The statistical behavior of the two-state exponential ON-OFF source is completely described by the following parameters: $\lambda_{O N}$, the rate of the Poisson process - in frames per second - during ON state; $\mu_{O N}$, the rate at which the underlying Markov process leaves ON state; $\mu_{O F F}$, the rate at which the Markov process leaves OFF state. The mean frame generation rate for the process is: $\lambda=\lambda_{O N} \cdot \frac{\mu_{O F F}}{\mu_{O N}+\mu_{O F F}}$. 
In Table I we present the three MMPP sources of the simulation study, in an increasing order with respect to burstiness. The simulation results for VoD, VDoP, MPR for the three sources of Table I are illustrated in Figures 2, 3, 4. Both VoD and VDoP curves for bursty MMPP sources move towards higher values compared to the less variable Poisson source of Fig. 1. Also we observe that the decline of MPR with TH is more aggressive with bursty sources (Fig. 4). As for that critical TH value, where monotonicities for VoD and VDoP change (start to increase), we observe that it moves towards the left (lower values) as the source burstiness decreases, and towards the right (higher values) for increasing burstiness. Assuming that the underlying arrival distribution does not change significantly with time, we can estimate the position of the critical TH value by using analytical or simulation results. The selection of a desirable TH value for the PVR will be a compromise between MPR and stream continuity. In all cases the TH value must reside on the left side of the critical TH value. It is in this area, that the decline of MPR is traded for better stream continuity. Moving to the right of the critical threshold value results in both worse MPR and worse stream continuity.

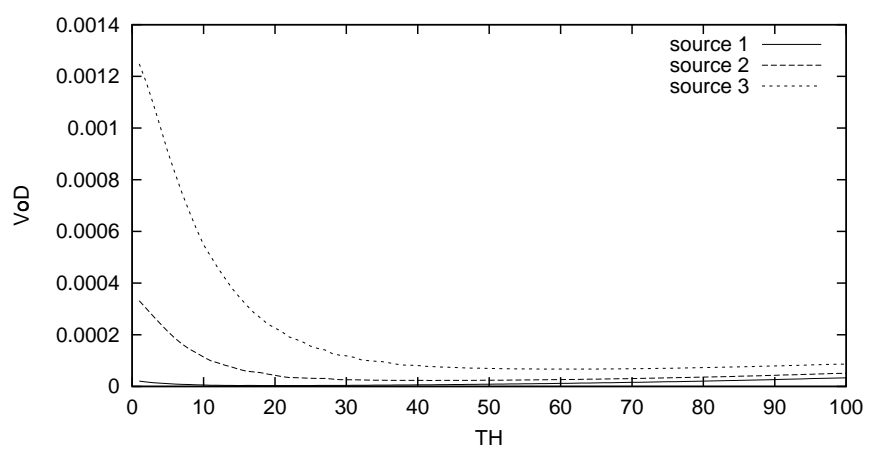

Fig. 2. VoD simulation results for MMPP sources of Table I. The three MMPP sources are in increasing order with respect to burstiness. Source 3 is the most bursty. VoD is in seconds ${ }^{2}$.

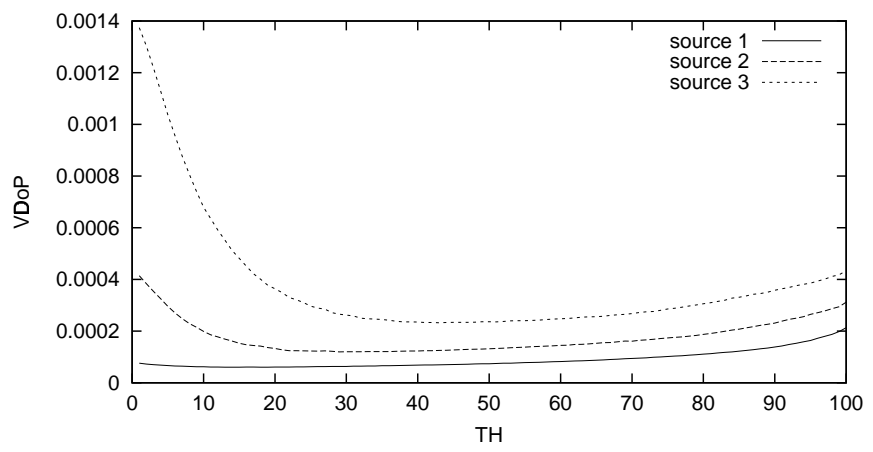

Fig. 3. VDoP simulation results for MMPP sources of Table I. VDoP is in seconds ${ }^{2}$.

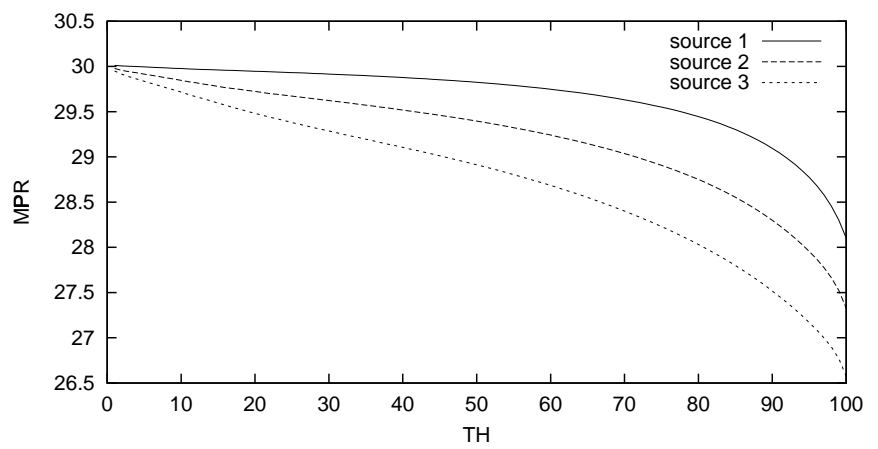

Fig. 4. MPR simulation results for MMPP sources of Table I. MRP is in frames/second.

\begin{tabular}{|c||c||c||c||c|}
\hline source & $\lambda_{O N}$ & $\mu_{O N}$ & $\mu_{O F F}$ & $\lambda$ (mean rate) \\
\hline 1 & 35 & 1 & 6 & 30 \\
\hline 2 & 40 & 1 & 3 & 30 \\
\hline 3 & 45 & 1 & 2 & 30 \\
\hline
\end{tabular}

TABLE I

MMPP SOURCES IN INCREASING ORDER WITH RESPECT TO BURSTINESS.

\section{Critical Threshold Online Detection Algorithm}

The critical threshold value $\left(\mathrm{TH}_{c}\right)$ that minimizes VDoP has been determined analytically, for Poisson arrivals (IV-A), and via simulation for MMPP sources (IV-B). Here, we present an online algorithm for the detection of $\mathrm{TH}_{c}$ when the underlying arrival process is an unknown MMPP or Poisson process ${ }^{2}$. The linear recursive filter [17] of (5),(6) is used for the online estimation of the values for VDoP, on a per frame basis. The estimates $V \hat{D} o P$ are averaged over a window $W$ to provide the smoothed estimate $\overline{V \hat{D} o P}$. Apart from being a sampling interval, $W$ is also the control interval for the $\mathrm{TH}_{c}$ detection algorithm. The algorithm looks for a minimum $\overline{V \hat{D} o P}$ by starting from an initial small value $\mathrm{TH}_{0}$ and increases the threshold by $\delta \mathrm{TH}$ every $W$ samples, until a change in the monotonicity of $\overline{V \hat{D o P}}$ is detected. The last threshold value serves as the estimate for $\mathrm{TH}_{c}$. We assume that the arrival process changes its statistical properties very slowly compared to the duration of the detection process. A sample implementation of the algorithm is presented in Table II. Fig. 7 illustrates the 2-level controller for the detection of $\mathrm{TH}_{c}$.

$$
\begin{gathered}
D \hat{o} P_{n} \triangleq a \cdot D o \hat{P}_{n-1}+(1-a) \cdot D o P_{n} \\
V \hat{D o} P_{n} \triangleq b \cdot V \hat{D o P_{n-1}}+(1-b) \cdot\left(D o P_{n}-D \hat{o} P_{n}\right)^{2}
\end{gathered}
$$

Fig. 5 illustrates the evolution of $V \hat{D} o P$ with time, for a PVR that starts with an initial threshold value $\mathrm{TH}=1$, and increments it in steps of $\delta \mathrm{TH}=10$ every $W=1000$ played frames. Frame interarrivals are generated by the first MMPP source of Table I. The linear recursive estimator uses $a=b=0.9999$. In Fig. 6 we plot $\overline{V \hat{D} o P}$, the corresponding smoothed version of $V \hat{D} o P$. The averaging over the window $W$ acts as a low pass filter on $V \hat{D} o P$. Running the online algorithm on these settings would return 21 as

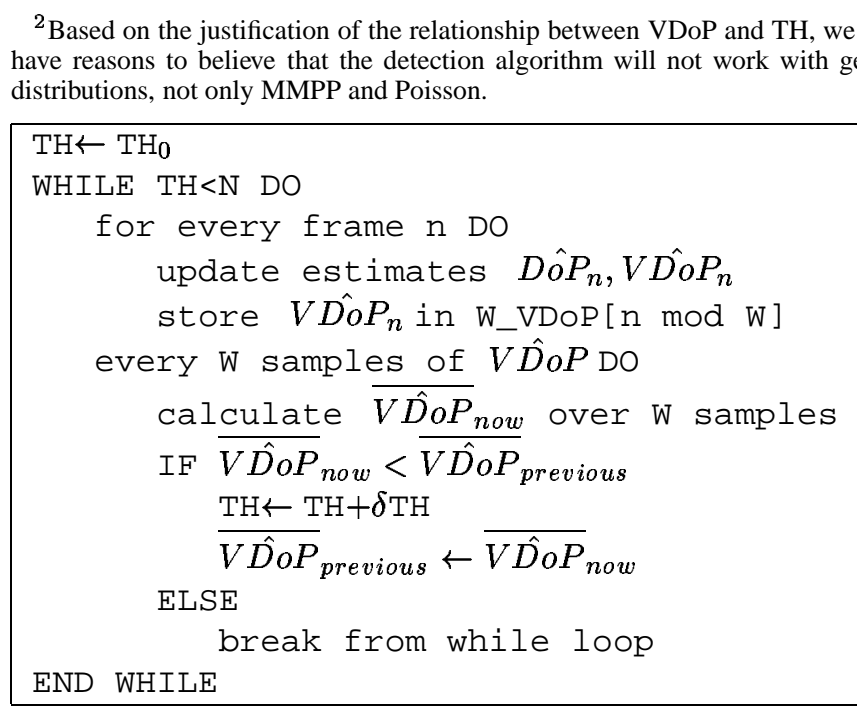

TABLE II

ONLINE ALGORITHM FOR THE DETECTION OF $\mathrm{TH}_{c}$ 


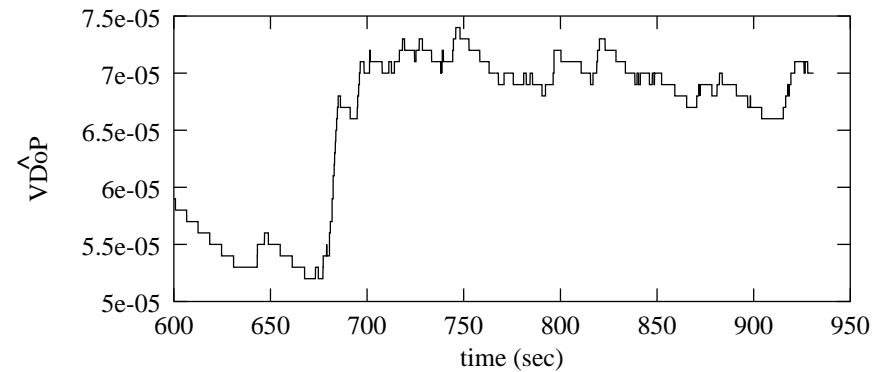

Fig. 5. $V \hat{D} o P$ plotted against time. TH is increasing from 1 to 91 in steps of $\delta \mathrm{TH}=10$ every $W=1000$ played frames. Interarrivals generated by the first MMPP source of Table I. The data presented are on a time window from 600 to 950 seconds following an initial warmup period.

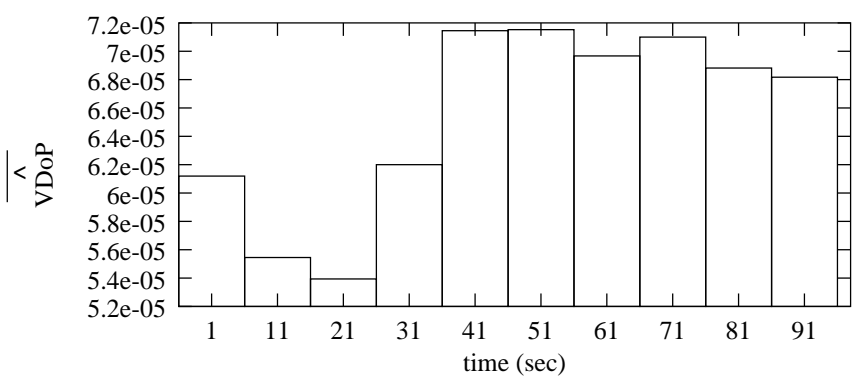

Fig. 6. $\overline{V \hat{D} o P}$ plotted against TH for the running $V \hat{D} o P$ estimation of Fig. 5.

The window mechanisms acts as a low pass filter on the $V \hat{D} \boldsymbol{o P}$ estimation.

the value of $\mathrm{TH}_{c}$. This is an accurate estimation for the optimal long term value of $\mathrm{TH}_{c}$ of Fig. 3 which is obtained by calculating VDoP (actual value) from four hour simulation runs. It must be noted that the scenario presented here is only to support the feasibility of the $\mathrm{TH}_{c}$ detection algorithm. For an actual implementation the choice of system parameters $(a, b, W, \delta \mathrm{TH})$ should be based on true interarrival measurements.

For the operation of the online algorithm for $\mathrm{TH}_{c}$, a static arrival process has been assumed during the detection phase. The value that will be determined by the algorithm is accurate for that specific arrival process. The arrival process however, can exhibit changes of its statistical behavior across various time scales. In this case the value for $\mathrm{TH}_{c}$ returned by the online algorithm can differ significantly from the real value for $\mathrm{TH}_{c}$ associated with the new arrival process. A control mechanism is required to identify the new critical threshold. Such a mechanism should monitor $\overline{V \hat{D} o P}$ and initiate a new search phase when the current $\overline{V \hat{D} o P}$ value differs from the $\overline{V \hat{D o P}}$ value of the initial $\mathrm{TH}_{c}$ more than a maximum distance $\epsilon$. The search for the new threshold $\mathrm{TH}_{c}^{\prime}$ would follow the paradigm of the detection algorithm, using a control interval $W$ and a step $\delta \mathrm{TH}$. The direction, relative to the current TH, in which the new detection phase should move can be determined from the understanding of the system behavior as revealed from Fig. 3, where $\mathrm{TH}_{c}$ moves towards higher values with increased interarrival variability. Consequently, a detection algorithm would compare the current $\overline{V \hat{D o P}}^{\prime}$ with the $\overline{V \hat{D} o P}$ of the initial $\mathrm{TH}_{c}$ and search for $\mathrm{TH}_{c}^{\prime}$ towards the left, if $\overline{V \hat{D} o P}^{\prime}<\overline{V \hat{D} o P}$ and towards the right, otherwise.

\section{CONCLUSIONS}

In this paper we have presented a playout adaptation algorithm for packet video receivers. The performance of the algorithm has been studied around two antagonistic performance metrics, the stream continuity and the reduction of mean playout rate. A new metric, the Variance of Distortion of Playout has been intro-

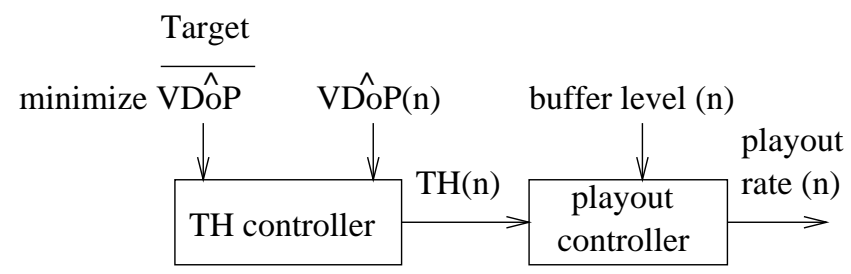

Fig. 7. 2-level controller for the detection of $\mathrm{TH}_{c}$

duced and its potential advantage over a previously used continuity metric has been pointed out. We have extended previous knowledge on continuity/playout-rate tradeoff, by identifying the area of threshold values, where a beneficial compromise between the two performance metrics exists. Based on analysis and simulation we have studied the behavior of the playout algorithm and its sensitivity to the variability of the arrival process. Based on that knowledge, we have proposed an online algorithm for the control of the rate adaptation mechanism in implemented systems, where the distribution of interarrivals can be allowed to change with time.

\section{REFERENCES}

[1] Reza Rejaie, Mark Handley, and Deborah Estrin, "An end-to-end rate-based congestion control mechanism for realtime streams in the internet," in IEEE INFOCOM, New York, Mar. 1999.

[2] Sally Floyd, Mark Handley, Jitendra Padhye, and Joerg Widmer, "Equationbased congestion control for unicast applications," in ACM SIGCOMM, Stockholm, Sweden, Aug. 2000, ACM.

[3] M. Yuang, Y. G. Chen, and C. L. Shen, "Dynamic video playout smoothing method for multimedia applications," in IEEE ICC, Dallas, Texas, June 1996, p. S44.

[4] Maria C. Yuang, Po L. Tien, and Shih T. Liang, "Intelligent video smoother for multimedia communications," IEEE J. Selected Areas Commun., vol. 15, no. 2, pp. 136-146, Feb. 1997.

[5] Kurt Rothermel and Tobias Helbig, "An adaptive stream synchronization protocol,” Durham, New Hampshire, Apr. 1995, Lecture Notes in Computer Science, pp. 189-202, Springer.

[6] William E. Naylor and Leonard Kleinrock, "Stream traffic communication in packet switched networks: destination buffering constraints," IEEE Trans. Commun., vol. COM-30, no. 12, pp. 2527-2534, Dec. 1982.

[7] Giulio Barberis and Daniele Pazzaglia, "Analysis and optimal design of a packet-voice receiver," IEEE Trans. Commun., vol. COM-28, no. 2, pp. 217 227, Feb. 1980.

[8] Ramachandran Ramjee, Jim Kurose, Don Towsley, and Henning Schulzrinne, "Adaptive playout mechanisms for packetized audio applications in widearea networks," in IEEE INFOCOM, Toronto, Canada, June 1994, pp. 680688, IEEE Computer Society Press, Los Alamitos, California.

[9] Sue B. Moon, Jim Kurose, and Don Towsley, "Packet audio playout delay adjustment: performance bounds and algorithms," ACM/Springer Multimedia Systems, vol. 5, no. 1, pp. 17-28, Jan. 1998.

[10] James D. Salehi, Zhi-Li Zhang, Jim Kurose, and Don Towsley, "Supporting stored video: Reducing rate variability and end-to-end resource requirements through optimal smoothing," IEEE/ACM Trans. Networking, vol. 6, no. 4, pp. 397-410, Aug. 1998.

[11] Z. Jiang and L. Kleinrock, "A general optimal video smoothing algorithm," in IEEE INFOCOM, San Francisco, California, March/April 1998, p. 676.

[12] Zhi-Li Zhang, Yuewei Wang, D. H. C. Du, and Dongli Su, "Video staging: A proxy-server-based approach to end-to-end video delivery over wide-area networks," IEEE/ACM Trans. Networking, vol. 8, no. 4, Aug. 2000.

[13] Jean-Chrysostome Bolot, "End-to-end packet delay and loss behavior in the Internet," in ACM SIGCOMM, Deepinder P. Sidhu, Ed., San Francisco, California, Sept. 1993, ACM, pp. 289-298, also in Computer Communication Review 23 (4), Oct. 1992.

[14] D. Sanghi, A. K. Agrawala, O. Gudmundson, and B. N. Jain, "Experimental assessment of end-to-end behavior on Internet," in IEEE INFOCOM, San Francisco, California, March/April 1993, pp. 867-874 (7d.2).

[15] Mark Claypool and Jonathan Tanner, "The effects of jitter on the perceptual quality of video," in ACM Multimedia '99, Orlando, FL, USA, 1999.

[16] W. Fischer and K. Meier-Hellstern, "The markov-modulated poisson process (MMPP) cookbook," Performance Evaluation, vol. 18, no. 2, pp. 149-171, 1993.

[17] Van Jacobson and Michael J. Karels, "Congestion avoidance and control," in ACM SIGCOMM. ACM, Nov. 1998. 